\title{
Larvas de Anisakidae na musculatura do pargo, Pagrus pagrus, no Estado do Rio de Janeiro, Brasil
}

Larval Anisakidae in musculature of Pagrus pagrus from the State of Rio de Janeiro, Brazil

\author{
Caroline D. R. Saad'; José L. Luque ${ }^{2 *}$ \\ ${ }^{1}$ Curso de Pós-Graduaçáo em Ciências Veterinárias, Universidade Federal Rural do Rio de Janeiro - UFRRJ, Bolsista do CNPq \\ ${ }^{2}$ Departamento de Parasitologia Animal, Instituto de Veterinária, Universidade Federal Rural do Rio de Janeiro - UFRRJ, \\ Bolsista de Produtividade de Pesquisa do CNPq \\ Recebido em 1 de Julho de 2008 \\ Aceito em 19 de Agosto de 2009
}

\begin{abstract}
Musculature of 36 specimens of Pagrus pagrus from the coastal zone of the State of Rio de Janeiro were examined to study larval of anisakid nematodes between January and May 2008. A total of 24 larval of Anisakis sp., Contracaecum sp., Hysterothylacium sp. and Raphidascaris sp. were collected infecting seven fishes (19.4\%). Larval Hysterothylacium sp. and Contracaecum sp. showed highest prevalence and Hysterothylacium sp. highest parasite abundance. This is the first record of larval anisakids in the somatic musculature of $P$. pagrus.
\end{abstract}

Keywords: Anisakidae, Nematoda, Pagrus pagrus, Rio de Janeiro.

\section{Resumo}

A musculatura de 36 espécimes de Pagrus pagrus, provenientes do litoral do Estado do Rio de Janeiro, foi examinada à procura de larvas de nematoides anisaquídeos entre janeiro e maio de 2008. Foi coletado um total de 24 larvas de Anisakis sp., Contracaecum sp., Hysterothylacium sp. e Raphidascaris sp. infectando sete peixes (19,4\%). Larvas de Hysterothylacium sp. e Contracaecum sp. mostraram a prevalência mais alta; e Hysterothylacium sp. o maior valor de abundância parasitária. Esse é o primeiro registro de larvas de anisaquídeos na musculatura somática de $P$. pagrus.

Palavras-chave: Anisakidae, Nematoda, Pagrus pagrus, Rio de Janeiro.

São escassos os trabalhos que mencionam a presença de larvas de nematóides anisaquídeos na musculatura somática de peixes marinhos do Brasil. A presença de larvas na musculatura, certamente, aumenta o potencial zoonótico desses parasitos que podem infectar o homem via ingestấo de carne de peixe crua ou insuficientemente cozida (ACHA; SZYFRES, 2003). Recentemente, relatos de Knoff et al. $(2004,2007)$ mencionaram a presença de anisaquídeos na musculatura de um peixe marinho (Genypterus brasiliensis) no Brasil, chamando ainda mais a atençáo para o potencial zoonótico dessas larvas por causa da sua localização no peixe.

O pargo Pagrus pagrus Linnaeus, 1758, é um peixe teleósteo marinho, de hábito demersal, que se alimenta principalmente de crustáceos, peixes e moluscos. Tipicamente bentopelágico, apresenta ampla e conhecida distribuição geográfica, incluindo a costa leste e oeste do Oceano Atlântico, Mar Mediterrâneo e ao norte das Ilhas Britânicas (MENEZES; FIGUEIREDO, 1980; HAIMOVICI et al., 1994). De ocorrência muito comum na costa sudeste-sul do Brasil, $P$. pagrus tem uma importância comercial bastante significativa, sendo pescado comercialmente entre o Espírito

\footnotetext{
*Autor para correspondência: José L. Luque

Departamento de Parasitologia Animal, Instituto de Veterinária,

Universidade Federal Rural do Rio de Janeiro - UFRRJ,

Rodovia BR-465, km 7, CP 74.508, CEP 23851-970 Seropédica - RJ,Brasil

e-mail: jlluque@ufrrj.br
}

Santo e o Rio Grande do Sul, no Brasil, servindo ao mercado interno e à exportação (YESAKI; BARCELLOS, 1974).

O pargo faz parte de um grupo de 44 espécies de peixes teleósteos marinhos do litoral do Estado do Rio de Janeiro com registro de larvas de anisaquídeos (TAVARES; LUQUE, 2006). Trabalhos realizados na mesma localidade por São Clemente et al. (1994) e Paraguassú et al. $(2000,2002)$ registraram esses parasitos na cavidade abdominal e vísceras de $P$. pagrus. As informaçôes fornecidas por esses autores mencionam altos valores de prevalência e intensidade parasitária dessas larvas, mas não apresentam informaçóes relacionadas com a sua presença na musculatura somática do hospedeiro. É importante mencionar, ainda, a patogenia provocada por larvas de anisaquídeos no fígado de P. pagrus coletado no litoral do Rio de Janeiro. Tal patogenia, identificada pela histopatologia da infecçáo, caracteriza-se por uma forte resposta celular do hospedeiro, com a presença de linfócitos, fibroblastos e fibras colágenas, que circundam essas larvas de nematóides na cápsula hepática (REGO et al., 1985; EIRAS; REGO, 1987).

Considerando-se a presença maciça de larvas de anisaquídeos registrada nas vísceras e órgáos de $P$. pagrus, o presente trabalho teve como objetivo desenvolver uma pesquisa voltada apenas para a musculatura somática desse peixe no litoral do Estado do Rio de 
Janeiro, para obter maiores subsídios para avaliação do potencial zoonótico dessas infecçóes.

No período de janeiro a maio de 2008, foram analisados 36 espécimes de $P$. pagrus. Os peixes, capturados no litoral do Estado do Rio de Janeiro, foram obtidos de um entreposto de pescado. Os espécimes encontravam-se congelados pelo processo prévio de congelamento rápido, em túnel de congelamento por ar forçado, onde o ar frio circula a uma velocidade de 3 a $5 \mathrm{~m} . \mathrm{s}^{-1}$ e a temperatura média no túnel é de $-35^{\circ} \mathrm{C}$, com uma umidade relativa do ar entre 60 e 70\% (BEVILACQUA et al., 2004), eviscerados, porém com as brânquias presentes. Uma vez obtidos, os peixes foram acondicionados em caixas de isopor contendo gelo, a fim de assegurar boas condiçôes para a coleta dos parasitos e protegê-los durante o transporte até o laboratório, na Universidade Federal Rural do Rio de Janeiro. Os peixes foram medidos e pesados. A média de peso dos espécimes foi de $0,398 \mathrm{~kg}(0,265$ a $0,525 \mathrm{~kg})$ e do comprimento total $28,2 \mathrm{~cm}(24 \mathrm{a} 34 \mathrm{~cm})$. Náo foi registrado o sexo dos hospedeiros, uma vez que foram obtidos eviscerados. $\mathrm{O}$ manual de peixes marinhos do Sudeste do Brasil de Menezes e Figueiredo (1980) foi referenciado para a respectiva identificação.

Para a coleta de parasitos, a musculatura somática dos peixes foi filetada a partir de uma incisão próxima aos opérculos até a inserção da nadadeira caudal, sendo inspecionada com auxílio de um estereomicroscópio e por transparência, utilizando-se um negatoscópio. Posteriormente, os parasitos foram fixados em Alcohol-Formalina-Ácido acético (A.F.A.) e preservados em etanol 70\%. A clarificação foi feita com Lactofenol de Amann e, eventualmente, com ácido acético glacial 100\%. A coleta, registro e processamento dos parasitos foram feitos de acordo com os procedimentos indicados por Eiras et al. (2006). A identificação das larvas de anisaquídeos foi feita por meio da chave dicotômica proposta por Tavares e Luque (2006). A prevalência, abundância e intensidade parasitárias foram calculadas de acordo com Bush et al. (1997). Espécimes representativos dos parasitos foram depositados na Coleção Helmintológica do Instituto Oswaldo Cruz, Rio de Janeiro, Brasil.

Dos 36 espécimes analisados, 7 (19,4\%) apresentavam larvas pertencentes a quatro gêneros de anisaquídeos: Anisakis sp., Contracaecum sp., Hysterothylacium sp. e Raphidascaris sp., encistadas na musculatura somática lateral e/ou ventral, sendo coletado um total de 24 larvas. Larvas de Hysterothylacium sp. e Contracaecum sp. apresentaram maior prevalência, e as de Raphidascaris sp. maior intensidade média parasitária (Tabela 1 ).

Larvas de anisaquídeos são comuns em muitas espécies de peixes marinhos do Rio de Janeiro (LUQUE; POULIN, 2004; TAVARES; LUQUE, 2006), porém não existem relatos sobre anisaquíase humana no Brasil. Essa doença pode ocorrer pela ingestáo da carne crua do pescado ou insuficientemente tratada pelo calor, salgada ou defumada, contendo larvas de terceiro ou quarto estágio. Nesse caso, o homem atua como um hospedeiro acidental, e as larvas não completam seu desenvolvimento, mas podem penetrar o trato digestório e invadir os órgãos anexos, provocando uma série de efeitos patológicos (LYMBERY; CHEAH, 2007).

A presença de larvas na musculatura dos peixes é própria de algumas espécies de anisaquídeos, como Anisakis simplex e Pseudoterranova decipiens, de reconhecida importância zoonótica, mas a presença de outras espécies de larvas de anisaquídeos, na musculatura somática, pode ser consequência de migração post-mortem ou durante o processo de congelamento (LYMBERY; CHEAH, 2007). No presente trabalho, as larvas encontravam-se todas mortas, fato que ressalta a importância do congelamento rápido em túnel de congelamento, o qual pode chegar a uma temperatura de $-35^{\circ} \mathrm{C}$. Tal temperatura, segundo a United States Food and Drug Administration (FDA), deve ser empregada num tempo de no mínimo 15 horas, ou ainda $-20{ }^{\circ} \mathrm{C}$, durante sete dias, para todo produto de pescado voltado para o consumo e preparado em temperaturas inferiores a $60{ }^{\circ} \mathrm{C}$ (FDA, 2008). Como as larvas de anisaquídeos são resistentes à salga, defumação e a procedimentos similares, a preparação do peixe para o consumo humano requer de temperaturas superiores aos $60{ }^{\circ} \mathrm{C}$ por pelo menos 10 minutos (SAKANARI; McKERROW, 1989). Entretanto, São Clemente et al. (1994) observaram em pargos infectados com anisaquídeos que, após 2 horas de exposição à temperatura de $-2,9^{\circ} \mathrm{C}$, todas as larvas coletadas encontravam-se mortas, embora seja importante mencionar que todas as larvas, neste estudo, estavam restritas às serosas das vísceras e serosas abdominais, o que pode indicar a necessidade de estudos mais específicos que, para diferentes espécies de peixes, podem resultar em protocolos diferentes de congelamento.

Knoff et al. (2007) salientaram que o meio mais eficaz para o controle da anisaquíase humana é a proibição da venda de peixes que não sejam submetidos aos processos descritos acima, frisando a importância da evisceração dos peixes imediatamente após

Tabela 1. Prevalência (P), abundância média (AM), intensidade média (IM) e local de infecção das larvas de anisaquídeos encontradas na musculatura somática de Pagrus pagrus do litoral do Estado do Rio de Janeiro.

\begin{tabular}{|c|c|c|c|c|}
\hline Parasitos & $\mathbf{P}(\%)$ & AM & IM & Local de infecção \\
\hline $\begin{array}{c}\text { Anisakis sp. } \\
\text { CHIOC No } 35663\end{array}$ & 5,56 & $0,06 \pm 0,24$ & 1,00 & musculatura lateral \\
\hline $\begin{array}{l}\text { Contracaecum sp. } \\
\text { CHIOC No } 35664\end{array}$ & 8,33 & $0,11 \pm 0,40$ & $1,33 \pm 1$ & musculatura lateral \\
\hline $\begin{array}{l}\text { Hysterothylacium sp. } \\
\text { CHIOC No } 35665\end{array}$ & 13,89 & $0,26 \pm 0,70$ & $1,80 \pm 0,84$ & $\begin{array}{c}\text { musculatura lateral } \\
\text { e ventral }\end{array}$ \\
\hline $\begin{array}{l}\text { Raphidascaris sp. } \\
\text { CHIOC No } 35666\end{array}$ & 5,56 & $0,17 \pm 0,86$ & $3,00 \pm 2,83$ & $\begin{array}{c}\text { musculatura lateral } \\
\text { e ventral }\end{array}$ \\
\hline Não identificados & 8,33 & $0,09 \pm 0,28$ & 1,00 & musculatura lateral \\
\hline
\end{tabular}


sua captura, para prevenir a possível migração das larvas para a musculatura. É bom lembrar que, no Brasil, existem dispositivos legais que consideram impróprio para consumo humano todo pescado que apresentar infestação muscular maciça (BRASIL, 1997).

O fato das larvas terem sido encontradas mortas na musculatura do pescado mostra também outra face do problema, pois se sabe que reaçóes alérgicas em humanos podem ocorrer com a ingestão de larvas mortas de anisaquídeos em razão da termoestabilidade dos alergênios presentes nestas e envolvidos nas respostas imunológicas, ressaltando a necessidade da realização de estudos para a identificação desses alergênios (TAVARES; LUQUE, 2006).

Tais consideraçóes mostram a relevância e a necessidade de estudos específicos, muito escassos no Brasil e na costa Atlântica da América do Sul, de detecção e identificação de parasitos com potencial zoonótico, principalmente localizados na musculatura do pescado, com o objetivo de prevenir a disseminaçáo de doenças parasitárias transmitidas por peixes.

\section{Referências}

ACHA, P. N.; SZYFRES, B. Zoonosis y enfermedades transmisibles comunes al hombre y a los animales. 3 ed. Washington: Organización Panamericana de la Salud, 2003. 544 p. (Publicación Científica y Técnica, n. 580).

BEVILACQUA, M.; D’AMORE, A.; POLONARA, F. A multi-criteria decision approach to choosing the optimal blanching-freezing system. Journal of Food Engineering, v. 63, n. 3, p. 253-263, 2004.

BRASIL. Decreto no 2.244, de 04 de junho de 1997. Altera dispositivos do Decreto no 30.691, de 29 de março de 1952, que aprovou o Regulamento de Inspeçáo Industrial e Sanitária de Produtos de Origem Animal (RIISPOA). Alterado pelo Decreto no 1.255, de 25 de junho de 1962, no 1.236 , de 2 de setembro de 1994 e no 1.812 , de fevereiro de 1996. Diário Oficial [da] República Federativa do Brasil, Poder Executivo, Brasília, DF, 05 de junho de 1997, Seção 1, p. 1155.

BUSH, A. O. et al. Parasitology meets ecology on its own terms: Margolis et al. revisited. Journal of Parasitology, v. 83, n. 4, p. 575-583, 1997.

EIRAS, J. C.; PAVANELli, G. C.; TAKEMOTO, R. M. Métodos de estudo e técnicas laboratoriais em parasitologia de peixes. 2 ed. Maringá: EDUEM, 2006. 199 p.

EIRAS, J. C.; REGO A. A. The histopathology of Scomber japonicus infection by Nematobothrium scombri (Trematoda: Didymozoidae) and of larval anisakid nematode infections in the liver of Pagrus pagrus. Memórias do Instituto Oswaldo Cruz, v. 82, n. 2, p. 155-159, 1987.

Food and Drug Administration - FDA. 2008. The bad bug book: a food borne pathogenic microorganisms and natural toxins handbook. Silver Spring: U.S. Food and Drug Administration. Disponível em: <http:// www.cfsan.fda.gov/ - mow/intro.html>. Acesso em: Junho 2008.
HAIMOVICI, M. et al. Demersal bony fish of the outer shelf and upper slope of the southern Brazil Subtropical Convergence Ecosystem. Marine Ecology Progress Series, v. 108, n. 1, p. 57-77, 1994.

KNOFF, M. et al. Primeira ocorrência de larvas de Anisakis sp. na musculatura de congro-rosa, Genypterus brasiliensis (Regan, 1903). Revista Brasileira de Ciência Veterinária, v. 11, n. 1/2, p. 119-120, 2004.

KNOFF, M. et al. Anisakidae parasitos de congro-rosa, Genypterus brasiliensis (Regan, 1903) comercializados no Estado do Rio de Janeiro, Brasil de interesse na saúde pública. Parasitologia Latinoamericana, v. 62, n. 3/4, p. 127-163, 2007.

LUQUE, J. L.; POULIN, R. Use of fish as intermediate hosts by helminth parasites: a comparative analysis. Acta Parasitologica, v. 49, n. 4, p. 353-361, 2004.

LYMBERY, A. J.; CHEAH, F. Y. Anisakid nematodes and anisakiasis. In: MURRELL, K. D.; FRIED, B. (Eds.). Food-Borne parasitic zoonoses: fish and Plant-Borne parasites. New York: Springer, 2007. p. 185-207. (World Class parasites, v. 11).

MENEZES, N. A.; FIGUEIREDO, J. L Manual de peixes marinhos do sudeste do Brasil. IV. Teleostei (3). São Paulo: Museu de Zoologia da Universidade de São Paulo, 1980. 96 p.

PARAgUASSÚ, A. R.; LUQUE, J. L.; ALVES, D. R. Aspectos quantitativos do parasitismo por larvas de anisakídeos (Nematoda: Ascaridoidea: Anisakidae) no pargo, Pagrus pagrus (Linaneus, 1758) (Osteichthyes: Sparidae) do litoral do Estado do Rio de Janeiro, Brasil. Contribuiçóes Avulsas Sobre a Historia Natural do Brasil, n. 24, p. 1-8, 2000.

PARAGUASSÚ, A. R.; LUQUE, J. L.; ALVES, D. R. Community ecology of metazoan parasites of red porgy Pagrus pagrus (L., 1758) (Osteichthyes: Sparidae) from the coastal zone of the State of Rio de Janeiro, Brazil. Acta Scientiarum, v. 24, n. 2, p. 461-467, 2002.

REGO A. A; CARVAJAL, J.; SCHAEFFER, G. Patogenia del hígado de peces (Pagrus pagrus (L.) provocada por larvas de nemátodos Anisakidae. Parasitología al Día, v. 9, n. 2, p. 75-79, 1985.

SAKANARI, J. A.; McKERROW, J. J. Anisakiasis. Clinical Microbiology Review, v. 2, n. 3, p. 278-284, 1989.

SÃO ClEMENTE, S. C.; UCHOA, C. M. A.; SERRA-FREIRE, N. M. Larvas de anisakídeos em Pagrus pagrus (Linnaeus, 1758) e seu controle através de baixas temperaturas. Revista Brasileira de Ciência Veterinária, v. 1, n. 1, p. 21-24, 1994.

TAVARES, L. E. R.; LUQUE, J. L. Sistemática, biologia e importância em saúde coletiva de larvas de Anisakidae (Nematoda: Ascaridoidea) parasitas de peixes ósseos marinhos do Estado do Rio de Janeiro, Brasil. In: SILVA-SOUZA, A. T. (Ed.). Sanidade de Organismos Aquáticos no Brasil. Maringá: Abrapoa, 2006. p. 297-328.

YESAKI, M.; BARCELLOS, B. N. Desenvolvimento da pesca do pargo-roseo ao largo da costa sul do Brasil. Rio de Janeiro: Programa de Pesquisa e Desenvolvimento Pesqueiro do Brasil, 1974. 18 p. (Documentos Ocasionais, n. 6). 